\title{
MECANISMOS FISIOLÓGICOS E FISIOPATOLÓGICOS DETERMI- NANTES DA ATIVIDADE VASOMOTORA SIMPÁTICA
}

\author{
PHYSIOLOGICAL AND PATHOPHYSIOLOGICAL MECHANISMS \\ DETERMINIG THE SYMPATHETIC VASOMOTOR TONE
}

\begin{abstract}
Abram Beutel ${ }^{1}$, Adilson M Silva ${ }^{1}$, Adriana PC Dugaich ${ }^{1}$, Ana Paula RL Galvão ${ }^{1}$, Bruno A Carillo ${ }^{1}$, Carolina R Barreto ${ }^{2}$, Elizabeth B de Oliveira-Sales ${ }^{1}$, Nayda P Abreu ${ }^{1}$, Pâmela BM Fontes ${ }^{1}$, Rafael S Carvalho', Raphael R de A. Freitas ${ }^{2}$, Miriam S Dolnikoff ${ }^{3}$, Cássia T Bergamaschi ${ }^{3}$, Ruy R Campos Jr. ${ }^{3}$
\end{abstract}

\begin{abstract}
${ }^{1}$ Aluno de Pós-Graduação. ${ }^{2}$ Aluno de Graduação. Universidade Federal de São Paulo.
${ }^{3}$ Docente. Disciplina de Fisiologia Cardiovascular e Respiratória. Escola Paulista de Medicina - UNIFESP.

Correspondência: Prof. Dr. Ruy R Campos

Disciplina de Fisiologia Cardiovascular e Respiratória - Escola Paulista de Medicina - UNIFESP.

Rua Botucatu - 862 CEP - 04023-060 São Paulo - SP
\end{abstract}

Beutel A, Silva AM, Dugaich APC, Galvão APRL, Carillo BA, Barreto CR, Oliveira-Sales EB, Abreu NP, Fontes PBM, Carvalho RS, Freitas RRA, Dolnikoff MS, Bergamaschi CT, Campos Jr. RR. Mecanismos fisiológicos e fisiopatológicos determinantes da atividade vasomotora simpática. Medicina (Ribeirão Preto) 2006; 39 (1): 65-76.

RESUMO: A atividade vasomotora simpática é um dos determinantes da pressão arterial (PA). Estabelecer quais são os mecanismos geradores dessa atividade é importante para o entendimento de como o sistema cardiovascular opera, tanto em situações fisiológicas como fisiopatológicas. Os principais grupos pré-motores do simpático estão confinados no núcleo paraventricular do hipotálamo (PVN) e região rostoventrolateral bulbar (RVLM). Em diversas situações fisiopatológicas há aumento na atividade vasomotora simpática, em parte conseqüente a maior atividade dos neurônios do PVN e RVLM. Nesta breve revisão, foram discutidos os principais mecanismos de ativação simpática em diferentes modelos experimentais: 1) hipertensão renovascular, 2) hipertensão por baixa massa renal, 3) insuficiência cardíaca, 4) hipertensão por bloqueio do óxido nítrico, 5) obesidade e 6) dimorfismo sexual. As ações de diferentes mediadores sobre o PVN e RVLM podem em longo prazo determinar novos patamares de atividade simpática, modificando os níveis tensionais e dessa forma, contribuir para a progressão da doença cardiovascular.

Descritores: Bulbo Ventrolateral. Óxido Nítrico. Pressão Arterial. Hipertensão. Insuficiência Cardíaca. Insuficiência Renal. Sistema Nervoso Simpático.

O sistema cardiovascular é o principal responsável pelo transporte de todas as substâncias necessárias ao bom funcionamento celular e a remoção dos produtos resultantes do metabolismo corporal. Para que isso se processe de forma adequada, a perfusão sanguínea tecidual e os níveis de pressão arterial (PA) precisam ser mantidos dentro de limites adequados. A ação integrada de diferentes sistemas de controle como, por exemplo, mecanismos neurais, hormonais, renais e locais é fundamental para a manutenção dos níveis ideais de PA e da perfusão tissular.

Dentre os vários sistemas de controle cardiovascular, os mecanismos neurais e hormonais têm despertado a atenção de diferentes grupos de pesquisa. O melhor entendimento de como tais sistemas atuam tanto a curto como em longo-prazo é relevante no que 
diz respeito ao avanço da fisiologia e da medicina e ao desenvolvimento de novas estratégias para o tratamento de doenças cardiovasculares.

O nosso conhecimento sobre os mecanismos de controle da atividade vasomotora simpática são relativamente recentes. Um grande impulso foi dado com a caracterização de um grupo restrito de neurônios localizados na porção ventral bulbar, hoje denominados de neurônios pré-motores do simpático da região rostroventrolateral (RVLM) ${ }^{1}$. Esses neurônios são fundamentais para a geração do tono vasomotor simpático, ou seja, a PA é controlada de momento a momento por esse grupo de células que se projetam monossinapticamente para a coluna intermédio-lateral, onde se localizam os neurônios pré-ganglionares do simpático.

Apesar de se reconhecer que a RVLM é uma região chave no controle cardiovascular, ainda se discute quais são os mecanismos geradores da atividade dos neurônios localizados nessa região. Duas hipóteses são as mais prováveis: 1) auto-ritmicidade, ou seja, os neurônios "cardiovasculares" da RVLM teriam atividade intrínseca do tipo marca-passo ${ }^{2}$ e, 2) teoria da rede de neurônios, segundo essa hipótese a atividade elétrica dos neurônios da RVLM seria comandada por potências pós-sinápticos excitatórios (PEPS). Aparentemente, essa ultima teoria é a mais provável ${ }^{3,4}$. Entretanto, em quais circunstâncias os neurônios da RVLM teriam atividade do tipo marca-passo, ainda precisam ser estabelecidas.

Parte da atividade da RVLM depende de uma pequena região localizada nas porções finais do bulbo ventrolateral, denominada de área pressora caudal (APC). Nosso laboratório tem se dedicado a estudar essa região. A inibição por GABA e glicina na APC produz quedas de pressão arterial de $30-40 \mathrm{mmHg}$ em ratos $^{5}$, e em coelhos ${ }^{6}$ e diminui a atividade simpática renal. Vários trabalhos demonstram que os neurônios da APC exercem uma atividade tônica sobre a manutenção da PA e atividade vasomotora ${ }^{7,8,9}$. Essa região aparentemente interfere na regulação da PA modulando a atividade dos neurônios da região caudoventrolateral (CVLM) e RVLM ${ }^{10}$.

Recentemente Sun e Panneton ${ }^{11}$ mostraram que os neurônios da APC se projetam para a CVLM e para a área A5, outra região de controle cardiovascular, sugerindo que estas vias seriam as responsáveis pelas respostas cardiovasculares mediadas pela APC.

Dados ainda não publicados do nosso laboratório mostram que em experimentos com ratos aneste- siados a inibição produzida pelo GABA e glicina na APC produz queda de PA por redução do débito cardíaco e da resistência periférica total, respostas semelhantes foram obtidas pela inibição da RVLM.

As funções fisiológicas exatas da APC ainda não são totalmente conhecidas. Além das ações cardiovasculares mediadas por essa região, alguns trabalhos sugerem a sua participação em mecanismos respiratórios em ratos acordados ${ }^{12}$ e provavelmente, nos ajustes cardiovasculares a estímulos nociceptivos ${ }^{11}$.

Acredita-se hoje que a atividade da RVLM em conjunto com outros núcleos pré-motores do simpático é fundamental para a manutenção do tono vasomotor simpático. O núcleo paraventricular do hipotálamo (PVN), por exemplo, é outra estrutura do sistema nervoso central (SNC) importante para o controle cardiovascular e manutenção da atividade vasomotora simpática. Essa atividade pode ser influenciada por diferentes sinalizadores e mediadores.

Não somente em situações fisiológicas, mas o estudo e o entendimento dos mecanismos envolvidos na geração da atividade vasomotora simpática e da PA em situações fisiopatológicas, pode trazer informações relevantes. Nosso laboratório tem se dedicado a estudar os mecanismos geradores e mantenedores da atividade simpática em diferentes situações experimentais. Nesta breve revisão abordaremos como esses mecanismos se processam, em alguns modelos experimentais. Dessa forma, abordaremos como a atividade simpática e a PA são reguladas e influenciadas nas seguintes situações: 1) hipertensão arterial experimental renovascular; 2) insuficiência renal crônica; 3) infarto do miocárdio; 4) obesidade; 5) bloqueio do óxido nítrico e 6) dimorfismo sexual.

\section{1- HIPERTENSÃO RENOVASCULAR E ATI- VAÇÃO SIMPÁTICA}

O modelo de hipertensão renovascular 2 Rins - 1 Clipe (2R-1C) foi criado por Goldblatt et al. ${ }^{13} \mathrm{e}$ consiste em estenose parcial da artéria renal de um dos rins. Vários estudos têm demonstrado em diferentes espécies, inclusive a humana, que o aumento dos níveis endógenos de angiotensina II (Ang II) em resposta à isquemia renal produz hipertensão arterial (HA) sustentada. Além disso, por ser considerado um modelo renina-dependente é amplamente estudado, uma vez que, aproximadamente $5 \%$ dos indivíduos hipertensos apresentam algum tipo de isquemia reno$\operatorname{vascular}^{14,15}$. 
Existem várias evidências de que o SNC está envolvido no desenvolvimento e manutenção da HA renovascular. O papel do sistema nervoso simpático (SNS) nas formas de HA secundárias como a HA renovascular passou a ser melhor entendido após o aparecimento de técnicas mais refinadas para medição de atividade simpática, como a liberação local (spillover) de noradrenalina, eletrofisiologia (registro direto de nervos simpáticos) e de imunohistoquímica ${ }^{16}$.

Head e Burke, em $2004^{17}$, pelo registro direto de nervo simpático renal, sugerem uma importante participação do SNS na manutenção da HA renovascular por meio de suas ações na liberação de renina e Ang II. Além disso, o SNS pode ainda regular a reabsorção de sódio e a resistência vascular renal, fatores que alteram a curva de função renal.

No modelo renovascular, existem evidências da participação de diferentes estruturas centrais no desenvolvimento da hipertonia simpática e HA. Na fase estável de hipertensão, há um aumento na atividade colinérgica da RVLM, sem nenhuma alteração na atividade de outros núcleos do bulbo, isto sugere, portanto, uma possível especificidade no aumento da atividade simpática e manutenção da hipertensão ${ }^{18}$.

Uma outra explicação para o aumento da atividade simpática é a ação central da Ang II nos tecidos periventriculares da região ântero-ventral do terceiro ventrículo $(\mathrm{AV} 3 \mathrm{~V})$ ou área postrema, regiões destituídas da barreira hemato-encefálica ${ }^{19,20}$.

Em estudo prévio desenvolvido em nosso laboratório, demonstrou-se que na HA renovascular há um aumento da atividade vasomotora simpática em parte por uma ação tônica de receptores glutamatérgicos localizados na RVLM, o bloqueio desses receptores produz queda da PA em ratos hipertensos renovasculares sem afetar os níveis tensionais em ratos contro$\operatorname{les}^{21}$. Trabalhos que relacionam a infusão de Ang II e a conseqüente liberação de glutamato na RVLM têm sido desenvolvidos e os resultados favorecem a hipótese de que a hipertonia glutamatérgica em resposta ao aumento crônico de Ang II pode ser um mecanismo importante no aumento do SNS e da $\mathrm{PA}^{22,23}$.

Outro recente achado em nosso laboratório foi que o bloqueio glutamatérgico central resulta numa menor resposta pressora a Ang II administrada perifericamente, sobretudo, quando baixas doses do peptídeo são administradas. $\mathrm{O}$ fato de a participação de receptores glutamatérgicos ter sido observada somente na dose mais baixa de Ang II deve-se provavelmente a hipótese de que nas doses altas do peptídeo, o efeito vasoconstritor periférico em receptores $\mathrm{AT}_{1}$ é máximo e sobrepuja as possíveis ações centrais do mesmo. Este resultado reforça a hipótese de que mecanismos glutamatérgicos centrais participam nas respostas cardiovasculares agudas à Ang II tanto a curto como em longo prazo.

Nos últimos anos, muitos trabalhos têm evidenciado o estresse oxidativo como um dos mecanismos envolvidos no desenvolvimento da HA renovascular. Os mecanismos geradores da atividade simpática podem ser desinibidos ou ainda estimulados pela ação dos superóxidos constituindo, portanto, um possível mecanismo para o aumento da atividade vasomotora simpática que acompanha a HA renovascular. Algumas evidências sugerem, por exemplo, que ao aumentar a formação de Ang II centralmente, um "feedback" positivo pode se estabelecer no qual a Ang II potencializa a sua própria formação, que por sua vez, aumenta a produção de superóxidos, sendo que tais fatores podem contribuir para a hipertonia simpática ${ }^{24}$. Entretanto, no modelo de hipertensão renovascular não se sabe exatamente qual o papel do estresse oxidativo nos mecanismos de controle cardiovascular ${ }^{25}$. Em estudo prévio em nosso laboratório, verificou-se que a administração endovenosa de ácido ascórbico (Vitamina C), um antioxidante, em animais hipertensos renovasculares, diminuiu a atividade do simpático renal e a PA logo após o início da infusão ${ }^{26}$. Dessa forma, é possível que a hipertonia simpática observada no modelo renovascular, parece resultar além de uma maior ação glutamatérgica central mediada pela Ang II, de componente dependente do estresse oxidativo.

\section{2- INSUFICIÊNCIA RENAL CRÔNICA E ATI- VAÇÃO SIMPÁTICA}

Um outro modelo que temos estudado no laboratório é a hipertensão por baixa massa renal. É sabido que a HA sistêmica é comum em pacientes com doença renal. Além disso, há uma hipertonia simpática associada à doença renal crônica. A isquemia renal, níveis elevados de Ang II circulante, a deficiência na produção de óxido nítrico (NO), o aumento da produção de espécies reativas de oxigênio (ROS) e a liberação de citocinas inflamatórias são provavelmente os principais responsáveis pelo aumento da atividade vasomotora simpática ${ }^{27}$. Entretanto, a participação específica de cada um desses fatores ainda não está esclarecida.

A isquemia renal é, provavelmente, o evento primário para o aumento da atividade simpática que é 
então seguida pelo aumento da $\mathrm{PA}^{28}$. Em modelos animais, a redução da massa renal expõe os néfrons remanescentes a mudanças hemodinâmicas adaptativas que inicialmente mantêm a função renal, mas que em longo prazo exercem um efeito deletério ${ }^{29}$.

Foi demonstrado que há uma ampla variedade de agressões glomerulares que resultam em elevada hipertensão glomerular, hipertensão sistêmica, permeabilidade glomerular alterada e proteinúria. Estas diversas patologias parecem incitar um ciclo vicioso de injúria e inflamação, resultando num padrão similar de nefropatia progressiva ${ }^{30}$.

A ativação de terminações nervosas simpáticas renais pode contribuir em longo termo para a elevação da PA. Sinais gerados em receptores sensoriais renais e conduzidos por aferências renais podem modificar a atividade eferente simpática com conseqüente aumento da $\mathrm{PA}^{31}$. A possibilidade de que o aumento crônico da atividade eferente simpática renal contribui para a HA tem sido defendida por vários autores $^{32}$. Como mencionado acima, a maior atividade simpática leva a maior liberação de renina e retenção de sódio, fatores que contribuem para o agravamento da doença renal.

Existem vários modelos de hipertensão de causa renal, entre eles, o modelo renovascular de Goldblatt 2R-1C e um rim-um clipe (1R-1C), o modelo com obstrução do ureter (UUO), ablação de massa renal e nefrectomia 5/6 entre outros. Entretanto, cada modelo tem características individuais e se comportam de forma específica frente à participação do SNS e do sistema renina-angiotensina-aldosterona (SRAA) no que diz respeito à manutenção dos níveis tensionais.

Estudos experimentais feitos em nosso laboratório utilizando-se ratos com nefrectomia 5/6 mostraram um aumento da atividade nervosa simpática renal quatro semanas após a cirurgia, seguida de um aumento significante da PA, o que condiz com os dados da literatura. Ratos com redução de massa renal (modelo 5/6) permanecem normotensos por apenas alguns dias após o procedimento cirúrgico, desenvolvendo HA em até seis dias após a cirurgia ${ }^{33}$. A hipertensão nessa fase é altamente dependente do sistema simpático, dados do nosso laboratório mostraram que a inibição da RVLM pela injeção de GABA nesses animais produz grande queda da PA, mais intensa do que a observada em animais não hipertensos.

Há um desequilíbrio entre baixa produção de NO, Ang II e atividade simpática aumentadas, caracterizando a doença renal. Todos esses fatores colabo- ram para um maior dano aos rins e ao coração, o que faz a doença renal chegar aos estágios finais levando a um pequeno índice de sobrevida aos pacientes quando não tratados adequadamente ${ }^{28}$. Dessa forma, estabelecer os mecanismos de sinalização que levam a hipertonia simpática nesses indivíduos será um importante avanço no entendimento da fisiopatogenia da insuficiência renal crônica.

\section{3- INSUFICIÊNCIA CARDÍACA CONGESTI- VA E ATIVAÇÃO SIMPÁTICA}

A ativação neuro-humoral é uma manifestação cardinal da insuficiência cardíaca congestiva (IC). O SRAA e o SNS são precocemente ativados após a isquemia produzida pela oclusão da artéria coronária descendente anterior esquerda gerando $\mathrm{IC}^{34}$.

A estratégia de tratamento mais eficiente aos portadores de IC é direcionada ao controle das manifestações periféricas que ocorrem em resposta à ativação neuro-humoral ${ }^{35}$. Atualmente, o fato de que os beta-bloqueadores tem sido utilizados com sucesso no tratamento da IC, é um exemplo de que a ativação simpática é deletéria no que diz respeito à progressão da doença. Entretanto, o entendimento dos mecanismos que levam à ativação dos mecanismos neurohumorais na IC é ainda limitado o que conseqüentemente limita as ferramentas terapêuticas.

Podemos considerar que as manifestações neuro-humorais encontradas na IC são teoricamente mediadas por mecanismos diferenciados quanto a sua origem, ou seja, de origem periférica ou central. Entretanto, a despeito dessa diferenciação, tais mecanismos interagem fortemente.

Na periferia, após o infarto do miocárdio, vários fatores vasoativos e neuroativos são liberados ${ }^{36}$. Dentre esses fatores, a Ang II e aldosterona, produtos do SRAA são ativados em resposta à baixa perfusão tissular. A ativação do SRAA contribui para aumentar a retenção de água e sódio, produzindo expansão do volume circulante e conseqüentemente maior sobrecarga ao coração, agravando a IC. A divisão entre fatores periféricos e centrais não é rigorosa, ou seja, a Ang II, pode também atuar centralmente causando ativação do simpático, que por sua vez, estimula a liberação de renina e também aumenta a reabsorção de sódio, fechando uma seqüência de eventos que perpetuam a ativação simpática e angiotensinérgica ${ }^{37}$. Adicionalmente, outro fator periférico importante é a ativação das citocinas pró-inflamatórias, produtos da 
ativação do sistema imunológico em resposta à injúria miocárdica ${ }^{38}$. Acredita-se que as citocinas atuam de forma efetiva, para produzir aumento da atividade simpática $^{39,40}$ e do SRAA ${ }^{41}$ e desta forma, também contribuem para o agravamento da IC. Os três fatores, SNS, SRAA e citocinas constituem provavelmente, os principais mecanismos geradores da disfunção autonômica que se instala com a progressão da IC.

Entre as respostas neuro-humorais encontradas na IC que claramente necessitam do envolvimento do SNC destacam-se: 1) aumento da sede e do apetite ao sódio ${ }^{42}$; 2) liberação do hormônio adrenocorticotrófico, com conseqüente aumento de corticosterona circulante $^{43}$;3) liberação de vasopressina ${ }^{44}$ e finalmente, 4) o aumento da atividade simpática ${ }^{45,46}$. O aumento da atividade simpática está diretamente relacionado ao remodelamento cardíaco; às arritmias cardíacas, e a vasoconstrição ${ }^{47}$. Como mencionado acima, a hipertonia do simpático caracteriza-se como um indicador de mau prognóstico na IC e, portanto, estabelecer quais são os mecanismos de ativação do simpático torna-se imperioso no tratamento de pacientes com IC.

Uma das primeiras evidências que correlacionaram modificações em regiões do $\mathrm{SNC}$ em reposta à IC foi obtida por Patel et al. ${ }^{48}$. Os autores demonstraram que os níveis de hexoquinase (um marcador de atividade metabólica) estavam aumentados no PVN em ratos submetidos à IC por infarto do miocárdio, quando comparados aos animais controles. Estes resultados foram confirmados posteriormente por VahidAnsari e Leenen ${ }^{49}$, em um estudo que utilizou como marcador a expressão da proteína Fos, um outro indicador de ativação neuronal em longo prazo, que também demonstrou a ativação do PVN nas fases crônicas da IC, sugerindo que a hipertonia simpática e as alterações hormonais possam ser em parte decorrentes do aumento na atividade deste grupo de neurônios pré-motores do simpático.

Grande parte dos neurotransmissores centrais é encontrada no PVN, dentre estes, o glutamato ${ }^{50}$ e a Ang $\mathrm{II}^{51}$ em geral, exercem efeitos excitatórios. Por outro lado, o GABA e o NO exercem influências inibitórias sobre a atividade simpática ${ }^{52}$. Desta forma, acredita-se que as interações e o balanço das ações excitatórias e inibitórias no PVN influenciam de forma significante a atividade vasomotora simpática durante o desenvolvimento da IC. Entretanto, os mecanismos exatos das interações excitatórias e inibitórias no PVN durante o desenvolvimento da IC não são totalmente conhecidos. Além dos neurotransmissores encontrados no PVN, podemos considerar que as ações da Ang II e citocinas (TNF- $\alpha$ ) são os principais candidatos envolvidos no desajuste autonômico que se estabelece à medida que a IC progride. Como cada um destes fatores, isoladamente contribui para a ativação simpática ainda não está bem estabelecido.

Além do PVN, outras regiões do SNC podem contribuir para a hipertonia simpática. Recentemente, DiBona e Jones ${ }^{53}$ demonstraram que existe um aumento na atividade de neurônios da RVLM em ratos com IC. Entretanto, não está totalmente estabelecido como este mecanismo é desencadeado. Uma das possibilidades é que o aumento da atividade da RVLM seja secundário ao aumento da atividade de neurônios do PVN, ou vice-versa, pois tais estruturas se comunicam intensamente.

Resultados de nosso laboratório demonstraram que há hipertonia simpática renal no modelo IC sem modificações no território esplâncnico, sugerindo, uma resposta ativadora simpática diferenciada. Esses resultados nos direcionam a novas abordagens experimentais com o intuito de caracterizar melhor a RVLM e o PVN, principais determinantes da atividade simpática, em animais com IC.

\section{4- OBESIDADE E ATIVAÇÃO SIMPÁTICA}

Estudos em humanos e em modelos experimentais têm demonstrado estreita associação entre intolerância à glicose, aumento da $\mathrm{PA}$, resistência à insulina/hiperinsulinemia, e dislipidemia, situação esta, atualmente referida como síndrome metabólica (SM) ${ }^{54,55}$. Indivíduos portadores da SM geralmente são obesos e constituem um grupo de alto risco para aquisição de doenças cardiovasculares ${ }^{56}$. A leptina (hormônio secretado pelos adipócitos) é secretada em grande quantidade, por ocasião da $\mathrm{SM}^{57}$ e parece ser um dos elementos de ligação entre a maior adiposidade e o aumento da atividade simpática cardiovascular. Sabe-se que, além dos efeitos sobre o apetite e o metabolismo, a leptina ao atuar no hipotálamo ventromedial e dorsomedial pode aumentar a atividade simpática renal bem como a $\mathrm{PA}^{58,59}$. Verificou-se inclusive que, pacientes obesos com hiperleptinemia apresentavam aumento da atividade simpática renal ${ }^{60}$. Essa ativação do simpático renal estimula a secreção de renina ${ }^{61}$, que ativa o SRAA. A aldosterona, bem como a ativação simpática direta sobre os túbulos renais provoca maior retenção de sódio contribuindo também para o aumento da PA. 
Em cães hipertensos por obesidade associada à $\operatorname{dieta}^{62}$, a denervação renal diminuiu a retenção de sódio bem como, o aumento da PA e isso reforça a idéia de que a ativação simpática renal desempenha importante papel no desencadeamento da hipertensão ${ }^{63}$. Resultados de nosso laboratório demonstraram que ratos normais tratados com sobrecarga de sacarose na dieta, por 30 dias, apresentam aumento na massa gordurosa, hiperleptinemia, hipertrigliceridemia e hipertensão. Como um dos mecanismos que participa na gênese desta hipertensão, nosso trabalho mostrou que há aumento na resistência vascular periférica e no tônus simpático. Além disso, há importante participação da Ang II. Estes resultados confirmam evidências anteriores da literatura nas quais, o bloqueio farmacológico do SRAA por inibidores da enzima conversora de angiotensina ou por antagonistas de receptores $\mathrm{AT}_{1}$ foi capaz de normalizar a PA bem como a captação periférica de glicose ${ }^{64,65}$. Neste modelo, a hiperinsulinemia parece também desempenhar papel importante no desencadeamento da hiperatividade simpática uma vez que, em ratos, demonstrou-se, nestas condições, ativação simpática para diferentes tecidos, incluindo o renal ${ }^{66}$. Em seres humanos, a administração de insulina e glicose também promove ativação do $\mathrm{SNS}^{67} \mathrm{e}$ em pacientes diabéticos, a infusão aguda de insulina aumenta a atividade simpática cardíaca, principalmente naqueles que já apresentam elevação da $\mathrm{PA}^{68}$. A ativação simpática na musculatura esquelética promove vasoconstricção e redução do fluxo sanguíneo prejudicando a captação periférica e promovendo, conseqüentemente, resistência à insulina ${ }^{69}$.

Recentemente, além da ativação simpática e do aumento na concentração plasmática de angiotensina, tem-se sugerido a participação do sistema angiotensinérgico tecidual como outro elemento envolvido nas alterações decorrentes da SM. Em adipócitos, o angiotensinogênio parece atuar localmente promovendo crescimento ou diferenciação dos mesmos além de poder ser também secretado para corrente sangüínea, contribuindo para o aumento do angiotensinogênio plasmático ${ }^{70}$. Esta observação sugere que, eventualmente, a Ang II tecidual possa desempenhar um importante papel tanto na gênese da obesidade como na hipertensão, que ocorrem na SM.

\section{5- ÓXIDO NÍTRICO E ATIVAÇÃO SIMPÁTICA}

O NO tem sido na última década alvo de vários estudos por exercer um importante papel na regulação do tono vasomotor e da PA em animais e huma- $\operatorname{nos}^{71,72,73}$. Sua liberação ocorre quando o aminoácido L-arginina é convertido em L-citrulina pela enzima óxido nítrico sintase (NOS) ${ }^{74,75}$. A disponibilidade do NO encontra-se reduzida na hipertensão essencial, sugerindo uma diminuição da vasodilatação nessa condição $^{76}$. Esses dados são reforçados por estudos que mostraram vasos em pacientes hipertensos uma redução na liberação de $\mathrm{NO}^{77,78}$.

Recentemente, pesquisas indicaram um importante papel do NO sobre a regulação da atividade simpática $^{79}$. Estudos em modelos experimentais mostraram que o NO apresenta-se como um regulador da atividade vasomotora e da PA, ao menos em parte, pela modulação da atividade simpática renal, pois em ratos, a injeção intravenosa do inibidor da NOS, a N ${ }_{-}$monomethyl-L-arginina (L-NMMA) produz aumento na PA e da atividade simpática renal ${ }^{80}$. Esse efeito excitatório simpático mostra-se aumentado após a denervação sinoaórtica, indicando que nesse modelo a ativação simpática encontra-se protegida em parte pelo barorreflexo ${ }^{81}$. Por outro lado, estudos em coelhos mostraram que o NO paradoxalmente pode exercer uma ação excitatória ${ }^{82}$. O NO derivado da enzima oxido nítrico sintase neuronal (nNOS), por exemplo, pode produzir aumento na $\mathrm{PA}^{83}$.

Dados da literatura mostram que a inibição aguda ou crônica do NO pode vir acompanhada de diferentes mudanças na atividade simpática ${ }^{75}$. Aparentemente, nos bloqueios crônicos a HA tem um importante componente simpático enquanto, nos bloqueios agudos não ${ }^{84,85,86}$. Resultados do nosso laboratório mostraram que a HA por bloqueio crônico do NO é totalmente dependente da atividade do SNS e que a RVLM é a origem da hipertonia simpática ${ }^{87}$. Comprovando a forte interação entre NO e simpático.

Há ainda aumento na produção de NO em situações de alto risco, como na sepse e no choque séptico, esse aumento deve-se em grande parte a ação de outras substâncias, como mediadores inflamatórios (incluindo o TNF $)^{88}$. As elevadas concentrações de NO são responsáveis pelas mudanças na atividade vasomotora, diminuição da PA e da função cardíaca, caracterizando a fase hipodinâmica da sepse ${ }^{89}$. A septicemia é caracterizada por uma resposta inflamatória generalizada tendo a influência do SNC como um sistema modulador ${ }^{90}$. Novos estudos têm se focado no papel do SNC em controlar a função da imunidade inata através da liberação de citocinas, já que na septicemia há intensa liberação das mesmas ${ }^{91,92}$. O SNS está intimamente envolvido no controle da inflamação e das respostas cardiovasculares associadas. No en- 
tanto, existem evidências de que o aumento na atividade parassimpática eferente e aferente constitui-se também num importante mecanismo protetor na sepse, o NO é um dos mediadores envolvidos ${ }^{93}$. Nosso laboratório tem se dedicado a estudar os mecanismos geradores da atividade simpática na condição de sepse, com ênfase em sinalizadores como o NO e TNF.

Assim, o NO desempenha um importante papel na modulação da atividade vasomotora simpática, tanto em situações normais como de hipertensão ou sepse. Portanto, além do NO produzir uma vasodilatação direta na vasculatura ele também pode inibir a atividade do SNS, promovendo assim, uma vasodilatação indireta.

\section{6- DIMORFISMO SEXUAL E ATIVAÇÃO SIM- PÁTICA}

Evidências indicam que a testosterona está envolvida no desenvolvimento e manutenção da HA. Esse hormônio masculino tem um papel fisiológico importante na regulação do sistema cardiovascular, visto que a incidência de HA é maior em homens do que em mulheres de mesma idade. Tais diferenças, também têm sido documentadas em vários modelos experimentais, como por exemplo, em ratos espontaneamente hipertensos (SHR), DOCA sal, Dahl salsensível, Sabra sal-sensível entre outros ${ }^{94,95,96}$.

Sabe-se que, a testosterona atuando diretamente no SNC pode resultar em aumento da $\mathrm{PA}^{97}$, pois o hormônio masculino é capaz de atravessar a barreira hemato-encefálica. Em ratas castradas o aumento de PA em resposta a testosterona é maior do que a observada em ratas não castradas, o aumento de PA é conseqüente à ativação do $\mathrm{SNS}^{98,99}$.

Foi também descrito, que o controle da função simpato-adrenal é diferente em fêmeas, particularmente, as vias regulatórias de liberação de adrenalina pelas supra-renais que parecem ser menos sensíveis aos estímulos excitatórios do que aos estímulos inibitórios em comparação aos machos, evidenciando o papel pressor da testosterona ${ }^{99,100}$.

Doenças cardiovasculares são a maior causa de morte em mulheres, e sua incidência é 4 vezes maior em mulheres pós-menopausadas do que em mulheres da mesma idade que estão na pré-menopausa ${ }^{101}$. A HA é o maior fator de risco para doença cardiovascular e, após ajuste para idade e índice de massa corpórea, as mulheres pós-menopausadas têm o dobro de chances de serem hipertensas quando comparadas às pré- menopausadas ${ }^{102}$. Dentre os mecanismos responsáveis pelo aumento da PA após a menopausa destacam-se: 1) alteração na relação entre estrógenos e andrógenos, 2) aumento no estresse oxidativo e nível endógeno de endotelinas, 3) ativação do SRAA, 4) ganho de peso e 5) ativação do $\mathrm{SNS}^{103}$.

A menopausa cirurgicamente induzida por ovarectomia em mulheres férteis e normotensas causa um aumento significativo na resistência vascular periférica e na PA, sugerindo um papel importante do estrógeno na homeostase cardiovacular ${ }^{104}$.

Alguns trabalhos demonstram que a proteção cardiovascular causada pelos estrógenos se faz através da modulação de vasoconstritores endógenos como a Ang II e vasodilatadores como o NO, além da redução dos níveis de lipoproteínas e colesterol ${ }^{105,106}$. O estrógeno é capaz de aumentar a biodisponibilidade de NO endotelial, que diminui a expressão do receptor $\mathrm{AT}_{1}$, promovendo vasodilatação ${ }^{107}$. Em camundongos, foi constatado a existência de dimorfismo sexual no que diz respeito a hipertensão crônica induzida por Ang II e que as fêmeas parecem estar protegidas desse aumento de PA provavelmente pelo estrogéno ${ }^{108}$.

Narkiewicz et al. ${ }^{109}$ afirmam que o envelhecimento é acompanhado por uma maior ativação simpática nas mulheres em relação aos homens, confirmando as ações protetoras dos estrógenos. A menor atividade do SNS pode contribuir para níveis crônicos mais baixos de PA em mulheres na pré-menopausa ${ }^{110}$.

Dados do nosso laboratório mostraram que o tratamento crônico com esteróide anabólico em ratos produziu HA, prejuízo no barorreflexo e ativação do SNS e SRAA. Em ratas, também observamos aumento nos níveis tensionais após a castração. A HA foi em ambos os casos, foi conseqüente a maior resistência vascular periférica.

Baseados nos estudos acima podemos afirmar que o dimorfismo sexual influencia a regulação barorreflexa da freqüência cardíaca e da PA. Os dados em conjunto, sugerem que a interação entre os esteróides gonadais e o SNS pode ser um importante mecanismo hipertensor.

\section{7- CONSIDERAÇÕES FINAIS}

Os mecanismos discutidos acima nessa breve revisão indicam que os sistemas geradores e mantenedores da atividade simpática e da PA são redundantes e altamente integrados. Diferentes sistemas podem ser ativados e desencadear um novo patamar 
de atividade vasomotora simpática, com conseqüente aumento nos níveis tensionais. A figura I mostra esquematicamente os principais mecanismos abordados nessa revisão. Os núcleos mais importantes em determinar a atividade vasomotora simpática, ou seja, o PVN e RVLM podem sofrer a ação de diferentes mediadores. $\mathrm{O}$ reconhecimento de como tais mecanismos operam é importante para melhor entender como o tono vasomotor simpático é gerado, tanto em situações fisiológicas como fisiopatológicas.

\section{Mecanismos de Ativaçāo Simpática}

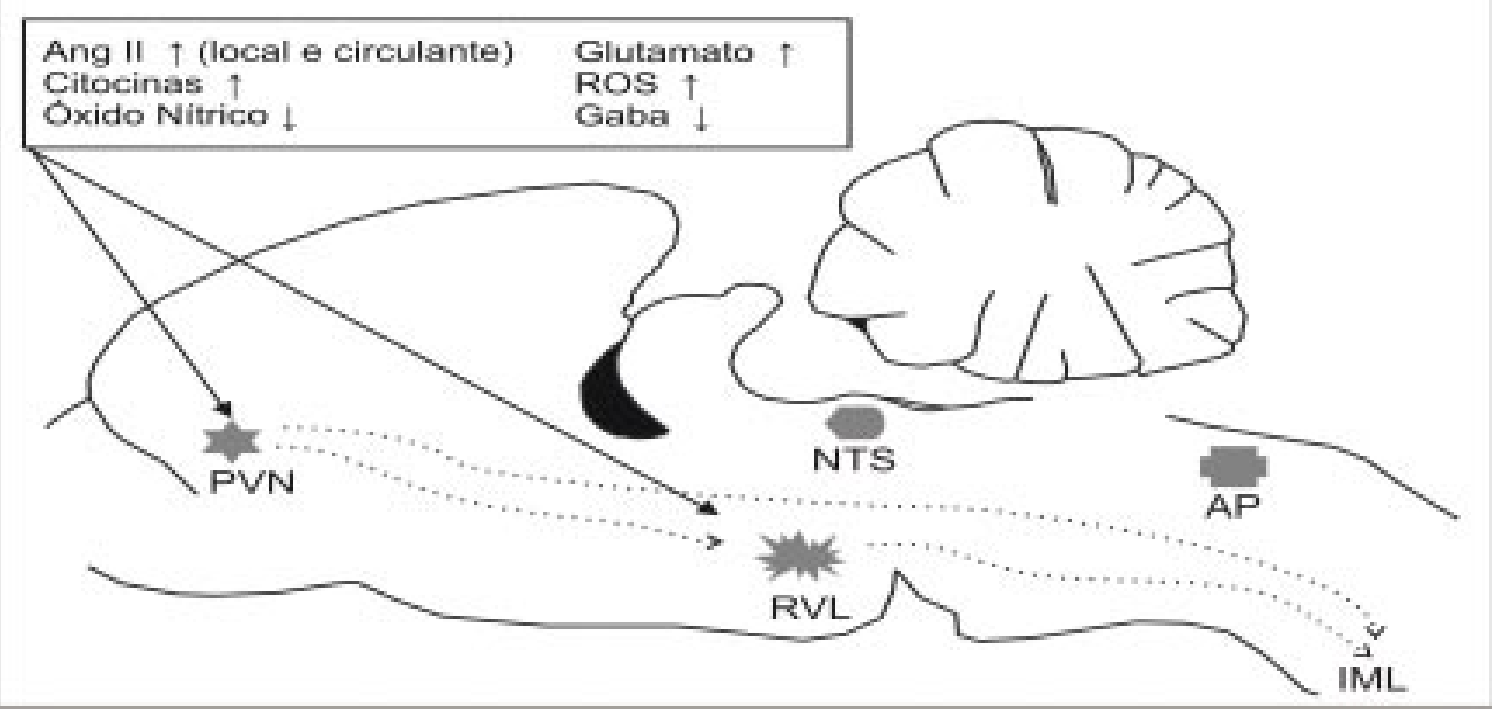

Figura 1: Representação esquemática de um corte sagital de cérebro de rato indicando a região rostroventrolateral do bulbo (RVLM) e o núcleo paraventricular do hipotálamo (PVN), ambos constituem-se núcleos pré-motores do simpático. As ações dos diferentes mediadores em tais núcleos podem determinar novos patamares de atividade vasomotora simpática. Núcleo do trato solitário (NTS); área postrema (AP); columa intermediolateral (IML).

Beutel A, Silva AM, Dugaich APC, Galvão APRL, Carillo BA, Barreto CR, Oliveira-Sales EB, Abreu NP, Fontes PBM, Carvalho RS, Freitas RRA, Dolnikoff MS, Bergamaschi CT, Campos Jr. RR. Physiological and pathophysiological mechanisms determinig the sympathetic vasomotor tone. Medicina (Ribeirão Preto) 2006;39 (1): 65-76.

ABSTRACT: The sympathetic vasomotor activity is one of determinants of blood pressure (BP). Understanding the mechanisms involved in the control of the cardiovascular system is important in physiological and pathophysiological condition. The principal sympathetic premotor brain nuclei are confined in the paraventricular nucleus of hypothalamus (PVN) and in the rostralventrolateral medulla (RVLM). In different patophysiological condition, there is an increase in the sympathetic vasomotor tone, in part due to an increase in the activity of the PVN and RVLM neurons. In this brief review, we discussed the major mechanisms of sympathetic activation in different experimental models: 1) renovascular hypertension, 2) renoprival hypertension, 3) cardiac failure, 4) hypertension induced by nitric oxide blockade, 5) obesity and 6) gender differences. The actions of different mediators in the PVN and in the RVLM acting in long term, can change the level of sympathetic nerve activity and blood pressure and therefore, contributing for the progression of cardiovascular disease.

Keywords: Ventrolateral Medulla. Nitric Oxide. Blood Pressure. Hypertension. Heart Failure. Renal Failure. Sympathetic Nervous System. 


\section{REFERÊNCIAS}

1 - Guertzenstein PG, Silver A. Fall in blood pressure produced from discrete regions of the ventral surface of the medulla by glycine and lesions. J Physiol (Lond) 1974; 242: 489-503.

2 - Guyenet PG. Role of ventral medulla oblongata in blood pressure regulation. In: Lowey AD, Spyer KM, eds. Central regulation of autonomic functions, New York: Oxford University Press; 1990. p. 145-67.

3 - Gebber GL. Central determinants of sympathetic nerve discharge. In: Lowey AD, Spyer KM, eds. Central regulation of autonomic functions, New York: Oxford University Press; 1990. p. $126-44$.

4 - Lipski J, Kanjhan R, Kruzewska B, Rong W. Properties of presympathetic neurones in the rostral ventrolateral medulla in the rat: an intracellular study "in vivo". J Physiol (Lond) 1996;90: 729-44.

5 - Campos Junior RR, Possas OS, Cravo SL, Lopes Ou , Guertzenstein PG. Putative pathways involved in cardiovascular responses evoked from the caudal pressor area. Braz J Med Biol Res 1994; 27(10): 2467-7.

6 - Dampney RA, Horiuchi J, Tagawa T, Fontes MA, Potts PD, Polson JW. Medullary and supramedullary mechanisms regulating sympathetic vasomotor tone. Acta Physiol Scand 2003; 177 (3): 209-18.

7 - Campos RR, Mcallen RM. Tonic drive to sympathetic premotor neurons of rostral ventrolateral medulla from caudal pressor area neurons. Am J Physiol 1999; 276(4 Pt 2): R 1209-13.

8 - Natarajan M, Morrison SF. Sympathoexcitatory CVLM neurons mediate responses to caudal pressor area stimulation. Am J Physiol Regul Integr Comp Physiol 2000; 279(2): R 364-74.

9 - Horiuchi J, Dampney RA. Evidence for tonic disinhibition of RVLM sympathoexcitatory neurons from the caudal pressor area. Auton Neurosci 2002;30;99(2): 102-10.

10 - Campos RR, Possas OSJ, Cravo SL, Lopes OU, Guertzenstein PG. In: Trouth CO, Millis RM, Kiwull-Schonn H, Schlafkepp $\mathrm{ME}$, eds. Ventral brainstem mechanisms and control of respiration and blood pressure. New York: Marcel Dekker; 1995. p 817-35.

11 - Sun W, Panneton WM. Defining projections from the caudal pressor area of the caudal ventrolateral medulla. J Comp Neurol 2005;482:273-93.

12 - Silva NF, Pires JG, Campos RR, Futuro Neto HA. Cardiovascular and respiratory responses to microinjection of L-glutamate into the caudal pressor area in conscious and anesthetized rats. Braz J Med Biol Res 2001, 34(12): 1603-6.

13 - Goldblatt H, Lynch J, Hanzal RF, Summerville WW. Studies on experimental hypertension. 1. The production of persistent elevation systolic blood pressure by means of renal ischemia. J Exp Med 1934;59: 347-78.

14 - Edmunds ME, Russel GI, Bing RF. Reversal of experimental renovascular hypertension. Hypertension 1991;9: 289-301.

15 - Melaragno MG, Fink G D. Slow pressor effect of angiotensin II in normotensive rats with renal artery stenosis. Clin Exp Pharmacol Phisiol 1996;23: 140-4.
16 - Grassi G. Counteracting the sympathetic nervous system in essential hypertension. Curr Opin Nephrol Hypertens 2004;13: 513-9.

17 - Head GA, Burke SL. Sympathetic responses to stress and rilmenidine in $2 \mathrm{~K} 1 \mathrm{C}$ rabbits. Evidence of enhanced nonvascular effector mechanism. Hypertension 2004;43: 636-42.

18 - Kubo T, Fukumori R, Kobayashi M, Yamaguchi H. Enhanced cholinergic activity in the medulla oblongata of DOCA-salt hypertensive and renal hypertension rats. Hypertens Res 1996;19: 213-9.

19 - Faber JE, Brody MJ. Neural contribution to renal hypertension following acute renal artery stenosis in conscious rats. Hypertension 1983;5 (Supp I): 155-64.

20 - Fink GD. Long term sympatho-excitatiry effect of angiotensin II: mechanism of spontaneous and renovascular hypertension. Clin Exp Pharmacol Physiol 1997;24: 91-5.

21 - Bergamaschi C, Campos RR, Schor N, Lopes OU. Role of the rostral ventrolateral medulla in maintenance of blood pressure in rats with Goldblatt hypertension. Hypertension 1995; 26: $1117-20$

22 - Katahira K, Mikami A, Otsuka A, Moriguchi A, Kohara K, Higaschimori K, Okuda M, Nagano M, Morishita R, Ogihara T. Differential control of vascular tone and heart rate by different amino acid neurotransmitters in the rostral ventrolateral medulla of the rat. Clin Exp Pharmacol Physiol 1994;21: 54556.

23 - Carvalho TH, Bergamaschi CT, Lopes OU, Campos RR. Role of endogenous angiotensin II on glutamatergic actions in the rostral ventrolateral medulla in Goldblatt hypertensive rats. Hypertension 2003,42: 707-12.

24 - Zanzinger J. Mechanisms of action of nitric oxide in the brain stem: role of oxidative stress. Auton Neurosci 2002; 98: $24-7$.

25 - De Champlain JO, Wu RO, Girouard HO, Karas MO, El Midaouia AO, Laplante MA, Wu L. Oxidative stress in hypertension. Clin Exp Hypertens 2004;26: 593-601.

26 - Oliveira-Sales EB, Dugaich AP, Abreu NP, Carillo B, Boim M, Bergamaschi CT, Campos RR. Oxidative Stress supports blood pressure and sympathetic activity in renovascular hypertension. FASEB J 2006. In Press.

27 - Koomans HA, Blankestijn PJ, Joles JA. Sympathetic hyperactivity in chronic renal failure: A wakeup call. J Am Soc Nephrol 2004;15: 524-37.

28 - Joles JA, Koomans HA. Causes and consequences of increased sympathetic activity in renal disease. Hypertension 2004;43: 699-706.

29 - Malvinder SP. Chronic renal disease. Br Med J 2002;325: 85-90.

30 - Remuzzi G, Bertani T. Pathophysiology or Progressive Nephropathies. N Engl J Med 1998;339: 1448-56.

31 - Grisk O, Retting R. Interactions between the sympathetic nervous system and the kidneys in arterial hypertension. Cardiovasc Res 2004;61: 238-46. 
32 - DiBona GF. The sympathetic nervous system and hypertension: Recent developments. Hypertension 2004;43: 147-50.

33 - Ye S, Mozayeni P, Gamburd M, Zhong H, Campese VM. Interleukin- $1 \mathrm{~b}$ and neurogenic control of blood pressure in normal rats and rats with chronic renal failure. Am J Physiol Heart Circ Physiol 2000;279: H2786-96.

34 - Francis J, Wei SG, Weiss RM, Felder RB. Brain angiotensinconverting enzyme activity and autonomic regulation in heart failure. Am J Physiol Heart Circ Physiol 2004;287: H2138-46.

35 - Middlekauff HR, Mark AL. The treatment of heart failure: the role of neurohumoral activation. Intern Med 1998;37: 112-22.

36 - Francis GS. Neurohumoral activation and progression of heart failure: hypothetical and clinical considerations. J Cardiovasc Pharmacol 1998;32: S16-S21.

37 - Felder RB, Francis J, Zhang ZH, Wei SG, Weiss RM, Johnson AK. Heart failure and the brain: new perspectives. Am J Physiol Regul Integr Comp Physiol 2003;284(2): R259-76.

38 - Frangogiannis NG, Smith CW, Entman ML. The in-flammatory response in myocardial infarction. Cardiovasc Res 2002;53: 31-47.

39 - Niijima A, Hori T, Aou S, Oomura Y. The effects of interleukin1 on the activity of adrenal, splenic and renal sympathetic nerves in the rat. J Auton Nerv Syst 1991;36: 183-92.

40 - Saindon CS, Blecha F, Musch TI, Morgan DA, Fels RJ, Kenney MJ. Effect of cervical vagotomy on sympathetic nerve responses to peripheral interleukin-1. Auton Neurosci 2001; 87: 243-8.

41 - Antonipillai I, Wang Y, Horton R. Tumor necrosis factor and interleukin-1 may regulate renin secretion. Endocrinology 1990;126: 273-8.

42 - Johnson AK, Thunhorst RL. The neuroendocrinology of thirst and salt appetite: visceral sensory signals and mechanisms of central integration. Front Neuroendocrinol 1997;18: 292-353.

43 - Catt KJ, Millan MA, Wynn PC, Mendelsohn FA, Aguilera G. Brain receptors for hypothalamic hormones. Adv Biochem Psychopharmacol 1987;43: 51-67.

44 - Mangiapane ML, Thrasher TN, Keil LC, Simpson JB, Ganong WF. Role for the subfornical organ in vasopressin. Brain Res Bull 1984;13(1): 43-7.

45 - Leenen FH. Cardiovascular consequences of sympathetic hyperactivity. Can J Cardiol 1999;15 (Suppl A): 2A-7A.

46 - Zucker IH, Wang W, Brandle M, Schultz HD, Patel KP. Neural regulation of sympathetic nerve activity in heart failure. Prog Cardiovasc Dis 1995;37: 397-414.

47 - Feuerstein GZ, Ruffolo RR Jr. Congestive heart failure and genomic medicine: a look into the 21st century. Cardiovasc Drugs Ther 1997;11(6): 713-7.

48 - Patel KP, Zhang PL, Krukoff TL. Alterations in brain hexokinase activity associated with heart failure in rats. Am J Physiol 1993;265: R 923-8.

49 - Vahid-Ansari F, Leenen FH. Pattern of neuronal activation in rats with CHF after myocardial infarction. Am J Physiol 1998;275: H2140-6.
50 - Bains JS, Ferguson AV. Reduced NMDA receptor sensitivity may underlie the resistance of subpopulations of PVN neurons to excitotoxicity. Neuroreport 1997;8: 2101-5.

51 - Pfister J, Spengler C, Grouzmann E, Raizada MK, Felix D, Imboden $\mathrm{H}$. Intracellular staining of angiotensin receptors in the PVN and SON of the rat. Brain Res 1997;754: 307-10.

52 - Li YF, Patel KP. Paraventricular nucleus of the hypothalamus and elevated sympathetic activity in heart failure: the altered inhibitory mechanisms. Acta Physiol Scand 2003;7 (1): 17-26.

53 - DiBona GF, Jones SY. Effect of dietary sodium intake on the responses to bicuculline in the paraventricular nucleus of rats. Hypertension 2001; 38(2): 192-7.

54 - Eaven GM. Role of insulin resistance in human disease. Diabetes 1988; 37: 1595-601.

55 - Lakka H-M, Laaksonen DE, Lakka TA, Niskanen LK, Kumpusalo E, Tuomilehto J, Salonen JT. The metabolic syndrome and total and cardiovascular disease mortality in middle-aged men. JAMA 2002; 21: 2709-16.

56 - Zimmet P, Alberti KGMM. The changing face of macrovascular disease in non-insulin dependent diabetes mellitus in different cultures: an epidemic in progress. Lancet 1997; 350 (Suppl. 1): S1-S4.

57 - De Courten M, Zimmet P, Hodge A. Hyperleptinaemia: the missing link in the metabolic syndrome? Diabetic Med 1997; 14: $200-8$.

58 - Carlyle M, Jones OB, Kuo JJ, Hall JE. Chronic cardiovascular and renal actions of leptin: role of adrenergic activity. Hypertension 2002; 39: 496-50.

59 - Marsh AJ, Fontes MA, Killinger S, Pawlak DB, Polson JW, Dampney RA. Cardiovascular responses evoked by leptin acting on neurons in the ventromedial and dorsomedial hypothalamus. Hypertension 2003;42: 488-93.

60 - Eikelis N, Schlaich M, Aggarwal A, Kaye D, Esler M. Interactions between leptin and the human sympathetic nervous system. Hypertension 2003;41: 1072-9.

61 - Young JB, Landsberg L. Catecholamines and the regulation of hormone secretion. Clin Endocrinol Metabol 1977; 6: 657-95.

62 - Hall JE. The kidney, hypertension, and obesity. Hypertension 2003; 41: 625-33.

63 - Kassab S, Kato T, Wilkins FC, Chen R, Hall JE, Granger JP. Renal denervation attenuates the sodium retention and hypertension associated with obesity. Hypertension 1995;25: 893-7.

64 - Navarro-Cid J, Maeso R, Perez-Vzcaino F, Cachoeiro V, Ruilope LM, Tamargo J, Lahera V. Effects of losartan on blood pressure, metabolic alterations, and vascular reactivity in the fructose-induced hypertensive rat. Hypertension 1995;26: 1074-8

65 - limura O, Shimamoto K, Matsuda K, Masuda A, Takizawa H, Higashiura K, Miyazaki Y, Hirata A, Ura N, Nakagawa M. Effects of angiotensin receptor antagonist and angiotensin converting enzyme inhibitor on insulin sensitivity in frutosefed hypertensive rats and essential hypertensives. Am J Hypertens 1995;8: 353-7. 
66 - Rahmouni K, Morgan DA, Morgan GA, Liu X, Sigmund CD Mark AL, Haynes WG. Hypothalamic PI3 kinase and MAP kinase differentially mediate regional sympathoactivation to insulin. J Clin Invest 2004; 114: 652-8.

67 - Rowe JW, Young JB, Minaker KL, Stevens AL, Palotta J Landsberg L. Effect of insulin and glucose infusions on SNS activity in normal man. Diabetes 1981;30: 219-25.

68 - Takagi M, Tanaka Y, Yamasaki Y, Yamamoto M, Hori M Nakaniwa T, Niwa M, Uchino H, Tamura Y, Nomiyama T, Watada H , Kawamori R. Responsiveness of insulin-induced cardiac sympathetic nerve activation associates with blood pressure regulation in diabetics. Am J Physiol Endocrinol Metab 2003;284: E1022-6.

69 - Landsberg L. Insulin and the sympathetic nervous system in the pathophysiology of hypertension. Blood Press 1996 (Suppl 1): 25-29.

70 - Massiera F, Bloch-Faure M, Ceiler D, Murakami K, Fukamizu A, Gasc JM, Quignard-Boulange A, Negrel R, Ailhaud G, Seydoux J, Meneton P, Teboul M. Adipose angiotensinogen is involved in adipose tissue growth and blood pressure regulation. FASEB J 2001; 15: 2727-9.

71 - Gokce N. L-arginine and hypertension. J Nutr 2004; 134 : 2807S-2811S, discussion 2818S-2819S.

72 - Haynes WG, Noon JP, Walker BR, Webb DJ. Inhibition of nitric oxide synthesis increases blood pressure in healthy humans. J Hypertens 1993; 11: 1375-80.

73 - Moncada S, Higgs A. The L-arginine-nitric oxide pathway. N Engl J Med 1993; 329: 2002-12.

74 - Palmer RM, Ashton DS, Moncada S. Vascular endothelial cells synthesize nitric oxide from L-arginine. Nature 1988; 333: 664-6.

75 - Ramchandra R, Barrett CJ, Malpas SC. Nitric oxide and sympathetic nerve activity in the control of blood pressure. Clin Exp Pharmacol Physiol 2005; 32: 440-6.

76 - Moss MB, Brunini TM, Soares De Moura R, Novaes Malagris LE, Roberts NB, Ellory JC, Mann GE, Mendes Ribeiro AC. Diminished L-arginine bioavailability in hypertension. Clin Sci (Lond) 2004; 107: 391-7.

77 - Camilletti A, Moretti N, Giacchetti G, Faloia E, Martarelli D, Mantero F, Mazzanti L. Decreased nitric oxide levels and increased calcium content in platelets of hypertensive patients. Am J Hypertens 2001; 14: 382-6.

78 - Surdacki A, Nowicki M, Sandmann J, Tsikas D, Boeger RH, Bode-Boeger SM, Kruszelnicka-Kwiatkowska O, Kokot F, Dubiel JS, Froelich JC. Reduced urinary excretion of nitric oxide metabolites and increased plasma levels of asymmetric dimethylarginine in men with essential hypertension. $\mathrm{J}$ Cardiovasc Pharmacol 1999; 33: 652-8.

79 - Sartori C, Lepori M, Scherrer U. Interaction between nitric oxide and the cholinergic and sympathetic nervous system in cardiovascular control in humans. Pharmacol Ther 2005; 106: $209-20$

80 - Sakuma I, Togashi H, Yoshioka M, Saito H, Yanagida M Tamura M, Kobayashi T, Yasuda H, Gross SS, Levi R. N ${ }^{G}$-methyl-Larginine, an inhibitor of $L$-arginine-derived nitric oxide synthesis, stimulates renal sympathetic nerve activity in vivo. $A$ role for nitric oxide in the central regulation of sympathetic tone? Circ Res 1992; 70: 607-11.

81 - Sakima A, Teruya H, Yamazato M, Matayoshi R, Muratani H, Fukiyama K. Prolonged NOS inhibition in the brain elevates blood pressure in normotensive rats. Am J Physiol 1998;275: R410-7.

82 - Hakim MA, Hirooka Y, Coleman MJ, Bennett MR, Dampney RA. Evidence for a critical role of nitric oxide in the tonic excitation of rabbit renal sympathetic preganglionic neurones. J Physiol 1995;82 (Pt 2): 401-7.

83 - Kurihara N, Alfie ME, Sigmon DH, Rhaleb NE, Shesely EG, Carretero OA. Role of nNOS in blood pressure regulation in eNOS null mutant mice. Hypertension 1998;32: 856-61.

84 - Sander M, Hansen J, Victor RG. The sympathetic nervous system is involved in the maintenance but not initiation of the hypertension induced by $\mathrm{N}$ (omega)-nitro-L-arginine methyl ester. Hypertension 1997;30: 64-70.

85 - Liu JL, Murakami H, Zucker IH. Angiotensin II-nitric oxide interaction on sympathetic outflow in conscious rabbits. Circ Res 1998;82: 496-502.

86 - Scrogin KE, Hatton DC, Chi Y, Luft FC. Chronic nitric oxide inhibition with L-NAME: effects on autonomic control of the cardiovascular system. Am J Physiol 1998; 274: R367-74.

87 - Bergamaschi CT, Biancardi VC, Lopes OU, Campos RR. Effects of angiotensin blockade in the rostral ventrolateral medulla on maintenance of hypertension induced by chronic L-NAME treatment. Brain Res 2002; 927: 195-9.

88 - Symeonides S, Balk RA. Nitric oxide in the pathogenesis of sepsis. Infect Dis Clin North Am 1999;13: 449-63.

89 - Balk RA. Severe sepsis and septic shock. Definitions, epidemiology, and clinical manifestations. Crit Care Clin 2000;16: 179-92.

90 - Tracey KJ. The inflammatory reflex. Nature 2002; 420(6917): 853-9.

91 - Tracey KJ, Vlassara H, CERAMI A.Cachetin/tumoe necrosis factor. Lancet 1989;1: 1122-6.

92 - Elenkov IJ, Wilder RL, Chrousos GP, Vizi ES. The sympathetic nerve-an integrative interface between two supersystems: the brain and the immune system. Pharmacol Rev 2000; 52: 595-638.

93 - Pavlov VA, Wang H, Czuza CJ, Friedman SG, Tracey KJ. The cholinergic anti-inflammatory pathway: a missing link in neuroimmunomodulation. Mol Med 2003; 9(5/8): 1 25-34.

94 - Share L, Crofton JT, Ouchi T. Vasopressin: sexual dimorphism in secretion, cardiovascular actions and hypertension. Am J Med Sci 1988; 295: 314-9.

95 - Reckelhoff JF, Zhang H , Granger JP. Testosterone exacerbates hypertension and reduces pressure-natriuresis in male spontaneously hypertensive rats. Hypertension 1998; 1 (1 Pt 2): 435-9.

96 - Khalid M, Ilhami N, Giudicelli Y, Dausse JP. Testosterone dependence of salt-induced hypertension in Sabra rats and role of renal á2-adrenoceptor subtype. J Pharmacol Exp Ther 2002; 300 (1): 43-9. 
97 - Beutel A, Bergamaschi CT, Campos RR. Effects of chronic anabolic steroid treatment on tonic and reflex cardiovascular control in male rats. J Steroid Biochem Mol Biol 2005; 93: $43-8$

98 - Esler MD, Lambert GW, Ferrier C, Kaye DM, Wallin BG, Kalff V, Kelly MJ, Jennings GL. Central nervous system noradrenergic control of sympathetic outflow in normotensive and hypertensive humans. Clin Exp Hypertens 1995; 17 (1/2): 409-23.

99 - Laborde CH, Chapa I, Lange D, Haywood JR. Gender differences in sympathetic nervous system regulation. Clin Exp Pharmacol Physiol 1999; 26: 122-6.

100 - Jones TJ, Dunphy G, Milsted A, Ely D. Testosterone effects on renal norepinephrine content and release in rats with different $Y$ chromosomes. Hypertension 1998; 32: 880-5.

101 - Kannel WB, Wilson PW. Risk factors that attenuate the female coronary disease advantage. Arch Intern Med 1995; 155: $57-61$.

102 - Tremollieres FA, Pouilles JM, Cauneille C, Ribot C. Coronary heart disease risk factors and menopause: a study in 1684 French women. Atherosclerosis 1999; 142: 415-23.

103 - Reckelhoff JF, Fortepiani LA. Novel mechanisms responsible for postmenopausal hypertension. Hypertension 2004; 43: $918-23$.
104 - Mercuro G, Zoncu S, Saiu F, Mascia M, Melis GB, Rosano GM. Menopause induced by oophorectomy reveals a role of ovarian estrogen on the maintenance of pressure homeostasis. Maturitas 2004; 47: 131-8.

105 - Dubey RK, Oparil S, Imthurn B, Jackson EK. Sex hormones and hypertension. Cardiovasc Res 2002; 53: 688-708.

106 - Mendelsohn ME, Karas RH. The protective effects of estrogen on the cardiovascular system. N Engl J Med 1999; 40(23): 1801-11.

107 - Harrison-Bernard LM, Schulman IH, Raij L. Postovarectomy hypertension is linked to increased renal $A T_{1}$ receptor and salt sensitivity. Hypertension 2003;42: 1157-63.

108 - Xue B, Pamidimukkala J, Hay M. Sex differences in the development of angiotensin Il-induced hypertension in conscious mice. Am J Physiol Heart Circ Physiol 2005; 288(5): $\mathrm{H} 2177-84$.

109 - Narkiewicz K, Phillips BG, Kato M, Hering D, Bieniaszewski L , Somers VK. Gender-selective interaction between aging, blood pressure, and sympathetic nerve activity. Hypertension 2005; 45: 522-5.

110 - Christou DD, Jones PP, Jordan J, Diedrich A, Robertson D, Seals DR. Women have lower tonic autonomic support of arterial blood pressure and less effective baroreflex buffering than men. Circulation 2005; 111: 494-8. 